\title{
Experimental observations of the universal cascade of bound states in quantum Ising chain in a magnetic field and $E_{8}$ symmetry
}

1. Exceptional $\mathbf{E}_{8}$ symmetry in spin dynamics of quasi-one-dimensional antiferromagnet $\mathrm{BaCo}_{2} \mathrm{~V}_{2} \mathrm{O}_{8}$ Authors: H. Zou, Y. Cui, X. Wang, Z. Zhang, J. Yang, G. Xu, A. Okutani, M. Hagiwara, M. Matsuda, G. Wang, G. Mussardo, K. Hódsági, M. Kormos, Z. Z. He, S. Kimura, Rong Yu, Weiqiang Yu, Jie Ma, Jianda Wu arXiv:2005.13302

2. Observation of $\mathbf{E}_{8}$ particles in an Ising chain antiferromagnet Authors: Z. Zhang, K. Amelin, X. Wang, H. Zou, J. Yang, U. Nagel, T. Rõõm, T. Dey, A. A. Nugroho, T. Lorenz, J. Wu, and Z. Wang Phys. Rev. B 101, 220411(R) (2020) [arXiv:2005.13772]

3. Experimental Observation of High-Energy $\mathrm{E}_{8}$ Particles in the Ising Chain Ferromagnet $\mathrm{CoNb}_{2} \mathrm{O}_{6}$

Authors: K. Amelin, J. Engelmayer, J. Viirok, U. Nagel, T. Rõõm, T. Lorenz, and Z. Wang

Phys. Rev. B 102, 104431 (2020) [arXiv:2006.12956]

Recommended with a Commentary by Masaki Oshikawa, Institute for Solid State Physics, University of Tokyo

A "coming of age" moment in learning physics might be when you realize that most models in physics cannot be solved exactly. Afterwards, theoretical physics looks like a pursuit of approximations. Then you have a refreshing encounter with nontrivial yet exactly solvable models in many-body physics, such as the Ising model on the two-dimensional square lattice and the $S=1 / 2 \mathrm{XXZ}$ chain. Although some of these models have experimental realizations (as good approximations) the fact remains that those models are very special. Nevertheless, the significance of the exactly solvable models is underscored by the notion of universality: critical behaviors of many systems belonging to the same "universality class" are essentially the same. Thus the exact solution of a very special model can describe the critical phenomena of many other models and real systems.

While the universality of critical phenomena is often introduced in terms of critical exponents, the concept of universality goes beyond the critical exponents. The long-distance 
asymptotic behaviors at the critical point are often described by a scale invariant (and often conformally invariant) field theory. Away from (but still close to) the critical point, the long-distance behaviors are described by a massive field theory. In fact, the scaling limit of statistical mechanical model near the critical point can be regarded as the definition of the field theory in the modern framework [1].

A delighting fact is that some of the continuum field theory is exactly solvable even if the underlying microscopic model is not. An impressive example is the $1+1$-dimensional Ising model under a (longitudinal) magnetic field. Onsager's celebrated exact solution of the twodimensional classical Ising model has been a source of inspiration for decades. The (quantum) Ising chain under a transverse magnetic field is equivalent to the two-dimensional classical Ising model, and is also exactly solvable. On the other hand, the two-dimensional classical Ising model in a magnetic field, or equivalently the quantum Ising chain under both the transverse and longitudinal field, is not exactly solvable, although the exact magnetization exponent has been known. Remarkably, Zamolodchikov [2] found that the scaling limit of these models near the quantum critical point is described by a factorized $S$-matrix of an integrable field theory. It turned out to be "affine Toda field theory" [3] associated with the $\mathrm{E}_{8}$ exceptional Lie algebra symmetry (for introduction to the mathematical aspects of $\mathrm{E}_{8}$, see Refs. $[4,5]$ ). That is, even though the Ising model under the longitudinal field is not exactly solvable, its scaling limit is described by an exactly solvable field theory with a surprisingly large emergent symmetry. This leads to numerous predictions beyond the critical exponents. Most importantly, existence of 8 stable quasiparticles with particular ratios of mass gaps is predicted. Physically, these quasiparticles can be interpreted as bound states of two domain walls. The lowest excitation energy needed to create each quasiparticle corresponds to the mass in the relativistic field theory. The $\mathrm{E}_{8}$ affine Toda field theory predicts exact ratios among these mass gaps. The ratio of two lowest masses is given by the golden ratio:

$$
\frac{m_{2}}{m_{1}}=\frac{1+\sqrt{5}}{2} .
$$

In fact, the two lowest-mass excitations were identified experimentally ten years ago in Ref. [6], which was highlighted in the Journal Club for Condensed Matter Physics [7]. However, a skeptic might still think that this single ratio can appear just by a coincidence.

The recent experimental works reported in the highlighted papers identify more quasiparticles, and found that they agree well with the theoretical prediction. This gives a more convincing evidence of the relevance of the $\mathrm{E}_{8}$ affine Toda field theory. Since two of the recent studies are on a different material than that was used in Ref. [6], this is also a good demonstration of universality. I refer the reader to Ref. [7] for more backgrounds, and focus on complementary issues in this commentary.

First let me comment about the materials. In the highlighted paper 3, $\mathrm{CoNb}_{2} \mathrm{O}_{6}$, which was also used in the earlier experimental demonstration of the $\mathrm{E}_{8}$ symmetry, is studied. While it has been regarded as a realization of the simplest theoretical model, namely the transversefield ferromagnetic Ising chain with $S=1 / 2$, it was recently pointed out that the system is more intricate [8]. Nevertheless, the quantum critical phenomena in one dimensional limit is still governed by the Ising universality class, and the $\mathrm{E}_{8}$ affine Toda field theory should remain a valid description once the interchain interaction is taken into account. 
In contrast, the material studied in the highlighted papers 2 and 3 is $\mathrm{BaCo}_{2} \mathrm{~V}_{2} \mathrm{O}_{8}$, which is a more complicated antiferromagnet. The phase diagram of $\mathrm{BaCo}_{2} \mathrm{~V}_{2} \mathrm{O}_{8}$ was studied in detail in Ref. [9]. According to it, $\mathrm{BaCO}_{2} \mathrm{~V}_{2} \mathrm{O}_{8}$ is rather modeled by coupled $S=1 / 2$ antiferromagnetic XXZ chains. The effective Hamiltonian of a single chain under the external magnetic field $H$ applied in $y$ direction is

$$
\begin{aligned}
\mathcal{H}_{X X Z}= & J \sum_{j}\left[\epsilon\left(S_{j}^{x} S_{j+1}^{x}+S_{j}^{y} S_{j+1}^{y}\right)+S_{j}^{z} S_{j+1}^{z}\right] \\
& -H \sum_{j}\left((-1)^{j} g_{x y} S_{j}^{x}+g_{y y} S_{j}^{y}+g_{y z} \cos \left(\frac{2 j-1}{4} \pi\right) S_{j}^{z}\right),
\end{aligned}
$$

where $\epsilon \sim 0.53$ is the anisotropy parameter and $g_{\alpha \beta}$ are the elements of the $g$-tensor. While the transverse interaction is sizable in $\mathrm{BaCo}_{2} \mathrm{~V}_{2} \mathrm{O}_{8}$, the longitudinal interaction along the easy axis is stronger. Thus, in the absence of the magnetic field, the ground-state has a long-range Néel order in z-component of the spins, breaking the $\mathbb{Z}_{2}$ (spin-flip) symmetry spontaneously.

When a magnetic field is applied in $y$ direction, the effective staggered field in $x$ direction proportional to $H g_{x y}$ induces quantum fluctuations in $z$-component of spins, playing the role of the transverse field in the Ising chain. As the applied field is increased, the spins are canted toward the effective staggered field, and the $\mathbb{Z}_{2}$ symmetry is eventually recovered for a strong applied field. Thus there should be a quantum phase transition at a critical field, and it turns out to be in the $(1+1)$-dimensional Ising universality class $[9,10]$. Concerning the critical behavior, the $y$ and $z$ components of the magnetic field are irrelevant.

In the presence of the long-range Néel order in z-components, the relatively weak but non-vanishing interchain interaction in the real material induces an effective staggered longitudinal field $\propto \sum_{j}(-1)^{j} S_{j}^{z}$ below the three-dimensional critical magnetic field. This plays the role of the longitudinal (uniform) magnetic field of the ferromagnetic transverse-field Ising chain. Therefore, despite the apparent differences, at the critical magnetic field of the pure one-dimensional model, the system is described by the same $\mathrm{E}_{8}$ affine Toda field theory as the critical transverse-field Ising chain in a small longitudinal field.

It is beyond the scope of the present commentary to review the $\mathrm{E}_{8}$ affine Toda field theory itself; the reader is referred to the comprehensive book [3] for details. Nevertheless, in the Appendix, I will review how the "magic numbers" representing the mass ratios can be obtained. It is a neat exercise which can be enjoyed even if you are not familiar with integrable field theories, or even with Lie algebras.

The three highlighted works identified several of these quasiparticle masses using different techniques: Terahertz $(\mathrm{THz})$ spectroscopy and inelastic neutron scattering. In either case, an incident photon or neutron creates an excited state, and its energy is resolved. At zero effective momentum transfer, a single-quasiparticle excitation gives rise to a delta-function peak (broadened by the experimental resolution) at the quasiparticle mass in the spectrum. It should be noted that the spectrum evolves continuously as the applied magnetic field is varied. It is when the effective transverse field is at the critical value (and the effective longitudinal field is the only relevant perturbation to the Ising conformal field theory), the $\mathrm{E}_{8}$ affine Toda field theory effectively describes the system. Thus the comparison to the $\mathrm{E}_{8}$ affine Toda field theory must be made for experimental data with the applied magnetic field 

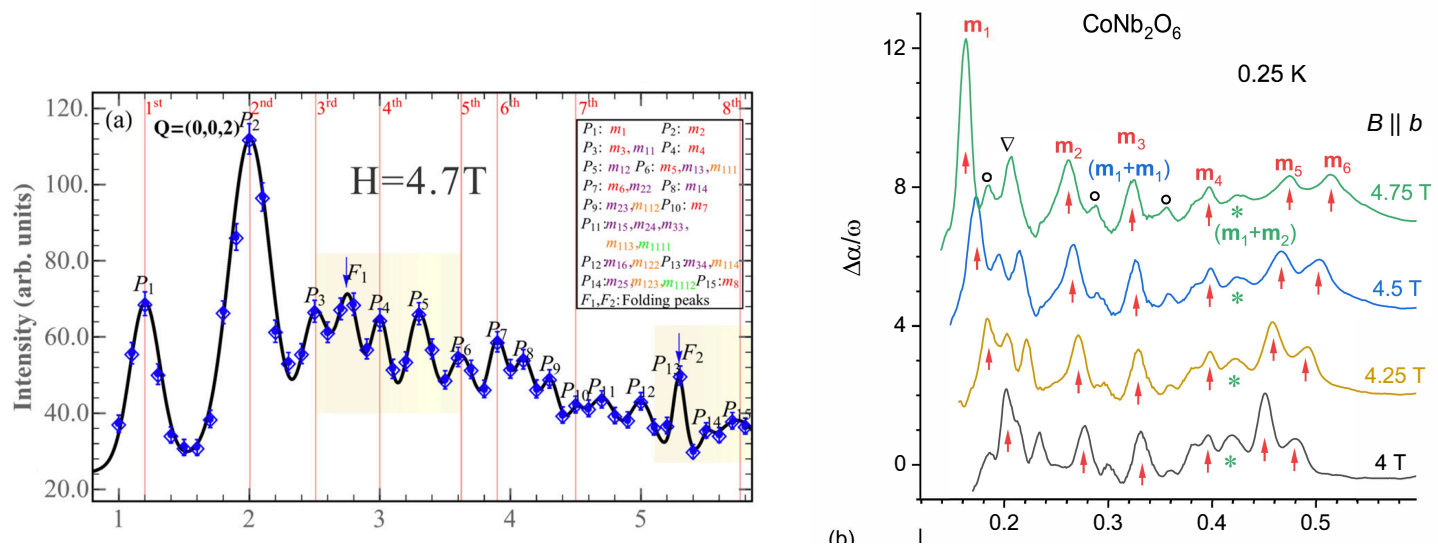

Figure 1: Experimental spectra detecting the $\mathrm{E}_{8}$ particles: inelastic neutron scattering intensity of $\mathrm{BaCo}_{2} \mathrm{~V}_{2} \mathrm{O}_{8}$ (left: from the highlighted paper 1), and optical absorption intensity of $\mathrm{CoNb}_{2} \mathrm{O}_{6}$ (right: from the highlighted paper 3 ).

corresponding to the $1 \mathrm{D}$ quantum critical point.

Remarkably, the peaks correspond to single quasiparticle masses predicted by the $\mathrm{E}_{8}$ affine Toda field theory are indeed observed up to $m_{5}$ (in the $\mathrm{THz}$ spectroscopy of $\mathrm{BaCo}_{2} \mathrm{~V}_{2} \mathrm{O}_{8}$ in the highlighted paper 2), $m_{6}$ (in the $\mathrm{THz}$ spectroscopy of $\mathrm{CoNb}_{2} \mathrm{O}_{6}$ in the highlighted paper 3 ), and possibly $m_{8}$ (in the neutron inelastic scattering in the highlighted paper 1, although the heaviest ones especially $m_{8}$ may be questionable), and the peak positions at the $1 \mathrm{D}$ quantum critical point agree well with the mass ratios from the $\mathrm{E}_{8}$ affine Toda field theory.

An important point is that, 5 quasiparticle masses $\left(m_{4}\right.$ and above) are heavier than $2 m_{1}$. In terms of energetics, such a quasiparticle can decay to two $m_{1}$ particles and thus is expected to be unstable. However, these heavier quasiparticles are protected from decaying by the symmetry and remain stable (within the $\mathrm{E}_{8}$ affine Toda field theory). Still you might expect that, the heavier quasiparticle peaks would be buried in the two-particle continuum (the continuum of the excited states with two quasiparticles with various relative momentum and zero total momentum). Somewhat surprisingly, however, the heavier quasiparticle peaks are rather clearly visible even in the two-particle continuum above $2 m_{1}$. This was predicted [11] by a numerical simulation using Time-Evolving Block Decimation (TEBD) and also supported by the recent theoretical calculations in the highlighted papers 1 and 2, based on the exact form factor of the $\mathrm{E}_{8}$ affine Toda field theory.

The further experimental confirmations of Zamolodchikov's remarkable theoretical prediction are not only impressive achievement of experimental magnetism but also demonstrate the power of universality, emergent symmetry, and emergent integrability. The author thanks Yuji Tachikawa for drawing his attention to the recent experimental works.

\section{References}

[1] John Cardy. The legacy of Ken Wilson. Journal of Statistical Mechanics: Theory and Experiment, 2013(10):P10002, oct 2013. 

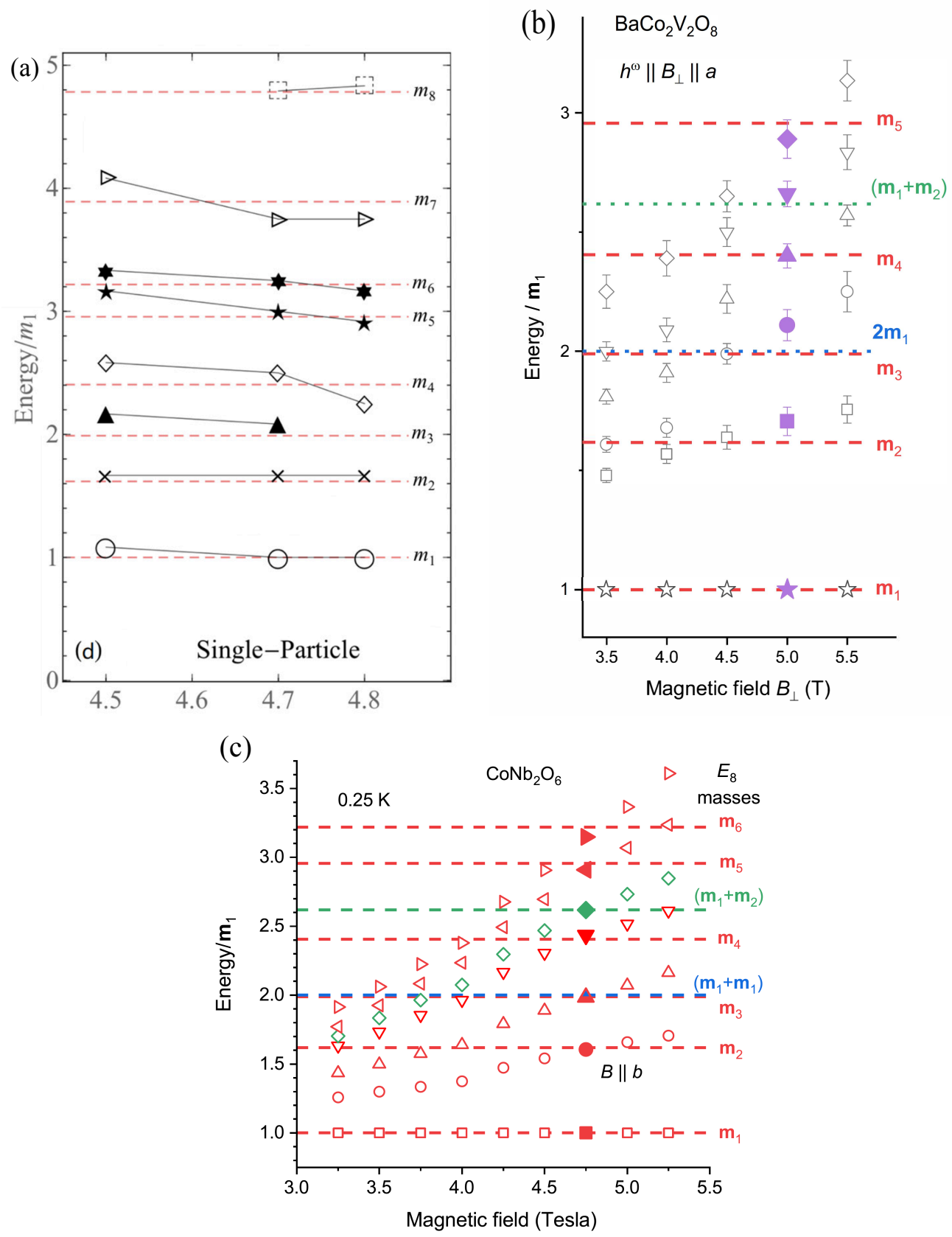

Figure 2: Experimentally identified masses (peak frequencies corresponding to singlequasiparticle creation) in unit of the smallest mass $m_{1}$. Panels (a), (b), and (c) are taken from the highlighted papers 1, 2, and 3, respectively. They agree well with the prediction of the $\mathrm{E}_{8}$ affine Toda field theory near the quantum critical point (corresponding to the applied field of $4.7 \mathrm{~T}$ to $5 \mathrm{~T}$ in both materials) of the one-dimensional system. 
[2] A. B. Zamolodchikov. Integrals of motion and $S$-matrix of the (scaled) $T=T_{c}$ Ising model with magnetic field. International Journal of Modern Physics A, 04(16):42354248, 1989.

[3] Giuseppe Mussardo. Statistical Field Theory. Oxford University Press, second edition, 2020.

[4] David Borthwick and Skip Garibaldi. Did a 1-dimensional magnet detect a 248dimensional Lie algebra? Not. Amer. Math. Soc., 58:1055-1066, September 2011.

[5] Skip Garibaldi. E8, the most exceptional group. Bull. Amer. Math. Soc. (N.S.), 53(4):643-671, 2016. arXiv:.

[6] R. Coldea, D. A. Tennant, E. M. Wheeler, E. Wawrzynska, D. Prabhakaran, M. Telling, K. Habicht, P. Smeibidl, and K. Kiefer. Quantum criticality in an Ising chain: Experimental evidence for emergent E E $_{8}$ symmetry. Science, 327(5962):177-180, 2010.

[7] Ashvin Vishwanath. Observation of universal bound state spectrum near a magnetic quantum critical point. Condensed Matter Physics Journal Club, March 2010, 2010.

[8] Michele Fava, Radu Coldea, and S. A. Parameswaran. Glide symmetry breaking and Ising criticality in the quasi-1D magnet $\mathrm{CoNb}_{2} \mathrm{O}_{6}$. arXiv e-prints, page arXiv:2004.04169, April 2020.

[9] Quentin Faure, Shintaro Takayoshi, Sylvain Petit, Virginie Simonet, Stéphane Raymond, Louis-Pierre Regnault, Martin Boehm, Jonathan S. White, Martin Månsson, Christian Rüegg, Pascal Lejay, Benjamin Canals, Thomas Lorenz, Shunsuke C. Furuya, Thierry Giamarchi, and Béatrice Grenier. Topological quantum phase transition in the Ising-like antiferromagnetic spin chain $\mathrm{BaCo}_{2} \mathrm{~V}_{2} \mathrm{O}_{8}$. Nature Physics, 14(7):716-722, Jul 2018.

[10] Shintaro Takayoshi, Shunsuke C. Furuya, and Thierry Giamarchi. Topological transition between competing orders in quantum spin chains. Phys. Rev. B, 98:184429, Nov 2018.

[11] Jonas A. Kjäll, Frank Pollmann, and Joel E. Moore. Bound states and $E_{8}$ symmetry effects in perturbed quantum Ising chains. Phys. Rev. B, 83:020407, Jan 2011.

[12] Howard Georgi. Lie Algebras In Particle Physics: from Isospin To Unified Theories. CRC Press, second edition, 2018. 


\section{Appendix: how to obtain the "magic numbers" (mass ratios in the affine Toda field theory)}

In this Appendix, I review (rather superficially) how the exact mass ratios in the $\mathrm{E}_{8}$ affine Toda field theory are obtained, based on the theory which had been developed long before the highlighted works in this commentary. While I do not give the logic behind the calculation (for that, the reader is referred to Ref. [3]), I describe the relatively simple procedure which could be followed with minimal backgrounds. I hope this helps the reader appreciate some part of the intriguing mathematics behind the remarkable prediction.

Lie algebras, which describe continuous symmetries, are classified in terms of Dynkin diagrams [12]. The Dynkin diagrams represents a geometric relation among "simple roots" of the Lie algebra. Here I focus on a class of Lie algebras called simply-laced Lie algebras. The Dynkin diagrams of the simply-laced Lie algebras are shown as in Fig. 3. There are two infinite series $\mathrm{A}_{n}$ and $\mathrm{D}_{n}$, which correspond to the special linear group $\mathrm{SL}(n)$ and the special orthogonal group in even dimensions $\mathrm{SO}(2 n)$. Besides them, there are three exceptional Lie algebras $\mathrm{E}_{6}, \mathrm{E}_{7}$, and $\mathrm{E}_{8}$. For each of these symmetries, there exists an integrable affine Toda field theory associated with the symmetry.

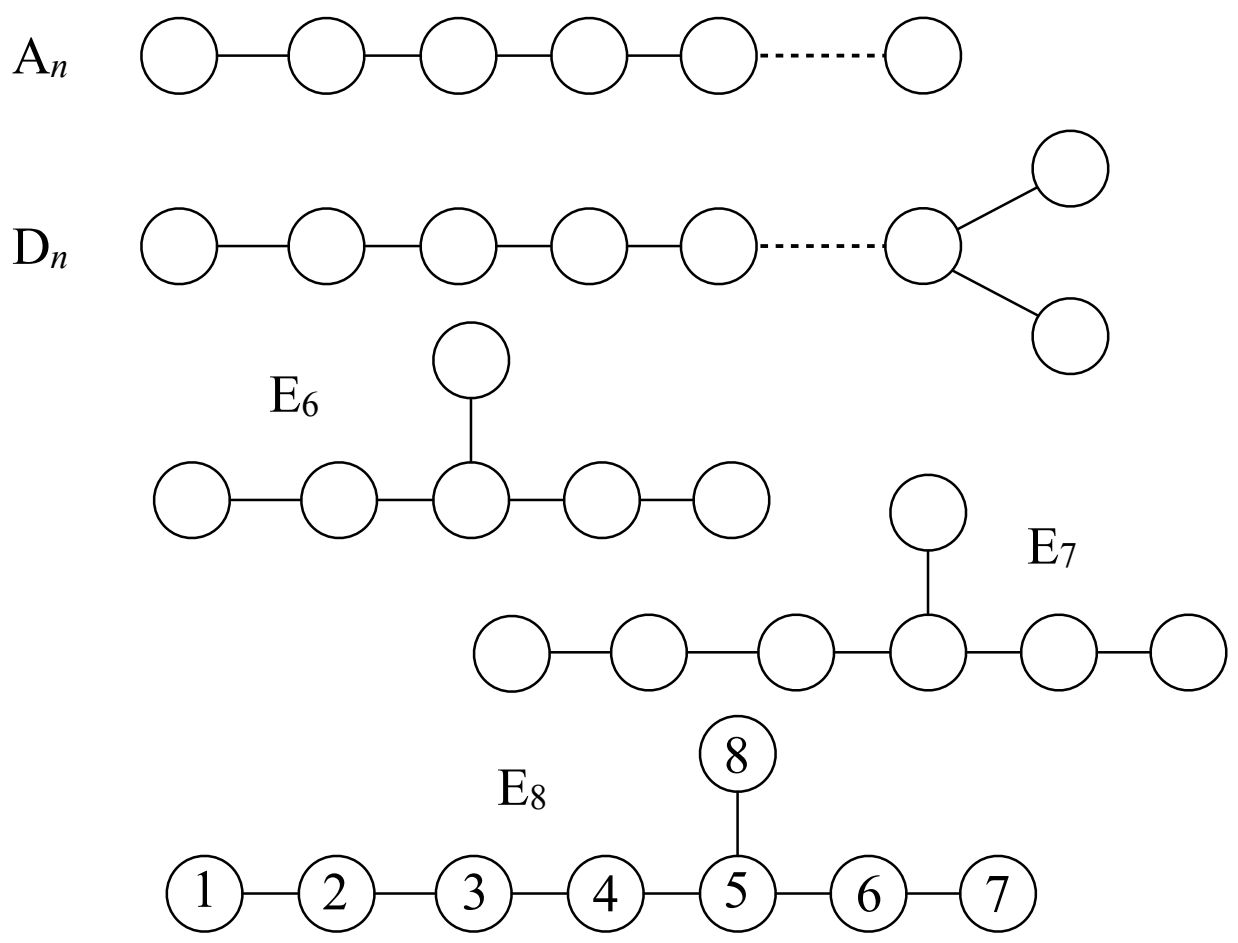

Figure 3: Dynkin diagrams of simply-laced Lie algebras. There are two infinite series $A_{n}$ $(n=1,2,3, \ldots)$ and $D_{n}(n=4,5,6, \ldots)$, and three exceptional Lie algebras $E_{6}, E_{7}$, and $\mathrm{E}_{8}$. The subscript represents the number of nodes. Each node of the Dynkin diagram for $\mathrm{E}_{8}$ is numbered for later convenience.

The Cartan matrix associated with the Dynkin diagram is given as 
- Each node in the Dynkin diagram corresponds to a basis of a vector space; the Cartan matrix $C$ is a square matrix of dimension equal to the number of nodes in the Dynkin diagram.

- Each diagonal element of $C$ is 2 .

- If the nodes $i, j$ are connected by a line, the matrix element $C_{i j}=-1$, while $C_{i j}=0$ if the two different nodes $i \neq j$ are not connected.

For example, the Cartan matrix for $\mathrm{E}_{8}$, for the numbering of the nodes as in Fig. 3, reads

$$
C=\left(\begin{array}{cccccccc}
2 & -1 & 0 & 0 & 0 & 0 & 0 & 0 \\
-1 & 2 & -1 & 0 & 0 & 0 & 0 & 0 \\
0 & -1 & 2 & -1 & 0 & 0 & 0 & 0 \\
0 & 0 & -1 & 2 & -1 & 0 & 0 & 0 \\
0 & 0 & 0 & -1 & 2 & -1 & 0 & -1 \\
0 & 0 & 0 & 0 & -1 & 2 & -1 & 0 \\
0 & 0 & 0 & 0 & 0 & -1 & 2 & 0 \\
0 & 0 & 0 & 0 & -1 & 0 & 0 & 2
\end{array}\right)
$$

The Cartan matrix can be interpreted as a tight-binding Hamiltonian on the Dynkin diagram with hopping amplitude -1 on every bond and the chemical potential 2 at every site. Let $\Psi$ be the ground state of the tight-binding Hamiltonian, namely

$$
C \Psi=E_{0} \Psi
$$

where $E_{0}$ is the ground-state energy (lowest eigenvalue of $C$ ). As in the case of the Schrödinger equation in the continuum, the ground-state is "nodeless" and every component is positive:

$$
\Psi_{j}>0
$$

More precisely, this is a consequence of the Perron-Frobenius theorem as the incidence matrix $2-C$ is a Perron-Frobenius matrix (with only non-negative elements and satisfying the connectivity condition). It turns out that these elements $\Psi_{j}$ of the "ground state wavefunction" give the mass spectrum of the affine Toda field theory associated with the Lie algebra [3]. Thus, the prediction of the mass ratios is reduced to obtaining the ground state of the tight-binding model on the Dynkin diagrams.

First let us consider the series $A_{n}$. The Dynkin diagram is just the linear chain of length $n$. The Cartan matrix then is the standard tight-binding model on a finite linear chain with uniform hopping matrix element and chemical potential with open boundary conditions. An eigenstate on the linear chain is given by a superposition of "plane waves" $e^{ \pm i p j}$ for a certain wavenumber $p$. In order to satisfy the open boundary conditions, which can be represented by $\Psi_{0}=\Psi_{n+1}=0$ with the extra fictitious sites 0 and $n+1$, the eigenstate should be

$$
\Psi_{j}=\sin \frac{\pi k j}{n+1},
$$

where $k=1,2, \ldots, n$, with the energy eigenvalue

$$
E=2-2 \cos \frac{\pi k}{n+1}
$$


The ground state is given by the smallest wavenumber $k=1$. Thus the mass spectrum is given by

$$
\Psi_{j}=\sin \frac{\pi j}{n+1},
$$

where $j=1,2, \ldots, n$.

Now let us move to the case of our interest, $\mathrm{E}_{8}$. The Dynkin diagram of $\mathrm{E}_{8}$ consists of linear chain segments, which shares the common site 5. Generalizing the analysis of $A_{n}$ above, on the chain segment consisting of sites 1 to 5 , the eigenstate can be written as

$$
\Psi_{j}=\sin p j(j=1,2,3,4,5),
$$

with a wavenumber $p$, which is undetermined at this point. The corresponding energy eigenvalue is

$$
E(p)=2-2 \cos p
$$

Similar argument applies to the shorter segments. For the sites 5, 6, and 7,

$$
\begin{aligned}
& \Psi_{6}=\Psi_{5} \frac{\sin 2 p}{\sin 3 p}=\frac{\sin 2 p \sin 5 p}{\sin 3 p}, \\
& \Psi_{7}=\Psi_{5} \frac{\sin p}{\sin 3 p}=\frac{\sin p \sin 5 p}{\sin 3 p},
\end{aligned}
$$

since we have already fixed $\Psi_{5}$ by Eq. (9). Similarly, for the sites 5 and 8,

$$
\Psi_{5}=\Psi_{5} \frac{\sin p}{\sin 2 p}=\frac{\sin p \sin 5 p}{\sin 2 p} .
$$

Here we need to use the same wavenumber $p$ for all $j$. So far $p$ is a free parameter, but we can fix it by requiring the eigenequation to be satisfied at site 5 :

$$
-\left(\Psi_{4}+\Psi_{6}+\Psi_{8}\right)+2 \Psi_{5}=E_{0} \Psi_{5}
$$

which implies

$$
\frac{\sin 4 p}{\sin 5 p}+\frac{\sin 2 p}{\sin 3 p}+\frac{\sin p}{\sin 2 p}=2 \cos p
$$

The solutions of this equation are

$$
\begin{aligned}
& p=\frac{k \pi}{30}, \\
& k=1,7,11,13,17,23,29 .
\end{aligned}
$$

The ground state is given by the smallest $p$ among them, $p=\pi / 30$. Then all the elements 
$\Psi_{j}$ are determined. If we order the masses by their magnitudes,

$$
\begin{aligned}
& m_{1}=\Psi_{1}, \\
& m_{2}=\Psi_{7}=\frac{1+\sqrt{5}}{2} m_{1} \sim 1.61803 m_{1}, \\
& m_{3}=\Psi_{2}=2 \cos \frac{\pi}{30} m_{1} \sim 1.98904 m_{1}, \\
& m_{4}=\Psi_{8}=\frac{1}{2 \sin \frac{\pi}{15}} m_{1} \sim 2.40487 m_{1}, \\
& m_{5}=\Psi_{3}=\frac{\sin \frac{\pi}{10}}{\sin \frac{\pi}{30}} m_{1} \sim 2.9563 m_{1}, \\
& m_{6}=\Psi_{6}=\frac{\sin \frac{\pi}{6} \sin \frac{\pi}{15}}{\sin \frac{\pi}{30} \sin \frac{\pi}{10}} m_{1} \sim 3.21834 m_{1}, \\
& m_{7}=\Psi_{4}=\frac{\sin \frac{2 \pi}{15}}{\sin \frac{\pi}{30}} m_{1} \sim 3.89116 m_{1}, \\
& m_{8}=\Psi_{5}=\frac{\sin \frac{\pi}{6}}{\sin \frac{\pi}{30}} m_{1} \sim 4.78339 m_{1} .
\end{aligned}
$$

This gives the desired mass spectrum in the $\mathrm{E}_{8}$ affine Toda field theory, which are experimentally "seen" in the highlighted works! The reader might want to try simular calculations for other (simply-laced) Dynkin diagrams and check them with Ref. [3]. 